\title{
Improvement in Successful Extubation in Newborns After a Protocol- driven Approach: A Quality Improvement Initiative
}

\author{
Rameshwar Prasad and Asit Kumar Mishra \\ From Department of Pediatrics, Tata Main Hospital, Jamshedpur, Jharkhand, India
}

Correspondence to: Dr Rameshwar Prasad, Flat no 3, Crystal Villa, Kadma Jamshedpur 831005 , Jharkhand, India. drrameshwarprasad@hotmail.com Received: June 22, 2018;

Initial review: December 03, 2018; Accepted: June 11, 2019.

\begin{abstract}
Objective: To reduce extubation failure rate by implementing protocol-driven ventilation and extubation strategies. Methods: Quality improvement project in a level II neonatal care unit from April 2017 to January 2018. Ventilation and extubation protocols implemented from 1 August, 2017. 18 ventilated newborns in the pre-protocol period, 16 in Plan-do-check-act (PDCA) cycle I and 17 in PDCA cycle II. Primary outcome was extubation failure within the first $72 \mathrm{~h}$ of extubation. Results: Extubation failure rate reduced from $41.7 \%$ (pre-protocol period) to $23.8 \%$ (PDCA 1 and 2 , OR $0.44,95 \% \mathrm{Cl} 0.12$ to $1.59, P=0.21$ ). Median time to first extubation attempt significantly decreased $(71.5 \mathrm{~h}$ to $38 \mathrm{~h}, P=0.046)$. Conclusions: A protocolized approach through quality improvement initiative demonstrated a sustained improvement in successful extubation with a significant reduction in the median time to first extubation attempt in ventilated newborns.
\end{abstract}

Keywords: Extubation failure, Neonate, Quality improvement, Ventilation.
$\mathrm{E}$ arly successful extubation of ventilated newborns is an important way to reduce ventilator-induced lung injury, sepsis and length of stay. Evidence-based strategies improve successful extubation rate $[1,2]$. Nonetheless, the inconsistent practices and arbitrary approach to ventilation and extubation result in repeated extubation failures and consequential reintubation $[3,4]$. An average of $25 \%$ ventilated newborns fail extubation $[5,6]$. Extremely preterm newborns have a much higher risk of failed extubation [7]. The evidence of protocol-driven management approach in reducing the duration of mechanical ventilation and weaning time is limited in newborns [8]. This study was designed to assess the impact of quality improvement on extubation failure in neonates.

\section{Methods}

The study was conducted in a level II neonatal care unit in an industrial hospital in India between 1 April, 2017 and 31 January, 2018. The study had 3 periods - Baseline (1 April, 2017 - 30 June, 2017), Plan-Do-Check-Act (PDCA) cycle I (1 August, 2017 - 31 October, 2017) and PDCA cycle II (1 November, 2017 - 31 January, 2018). Our primary outcome was extubation failure which was defined as reintubation within 72 hours of extubation irrespective of the cause [6]. Reintubation was defined as intubation occurring after 72 hours of extubation and was excluded from extubation failure. During the baseline period, patient data were collected by reviewing patients' charts and electronic database. We constituted a team comprising a consultant-in-charge of the neonatal unit, specialists, a neonatologist and two senior nursing staff. In a brainstorming session, the probable causes of extubation failure were identified and put into a fishbone diagram (Fig. 1). Most of the modifiable root causes of extubation failure belonged to man and method groups. Further, why-why analysis led us to the two main problems of lack of standardized ventilation and extubation strategies.

We addressed the above two problems by drafting ventilation and extubation strategies by searching the literature for evidence-based practices. We prepared a checklist for extubation readiness. Printed copies of ventilator strategy, ventilator-associated pneumonia (VAP) prevention bundle and checklist for extubation readiness were displayed at the bedside. Training was given to residents, pediatricians and staff nurses twice a month about the protocol and records maintained. The consultant-in-charge of the neonatal unit crosschecked the extubation checklists before taking a decision to extubate a newborn. Post-extubation adrenaline nebulization was given regularly although its evidence is lacking in newborns. We did not include peri-extubation dexamethasone in the protocol due to concerns over both short and long term complications.

The protocols were implemented from 1 August, 2017. Ethical approval was not sought as this was a quality improvement initiative. Our primary objective 


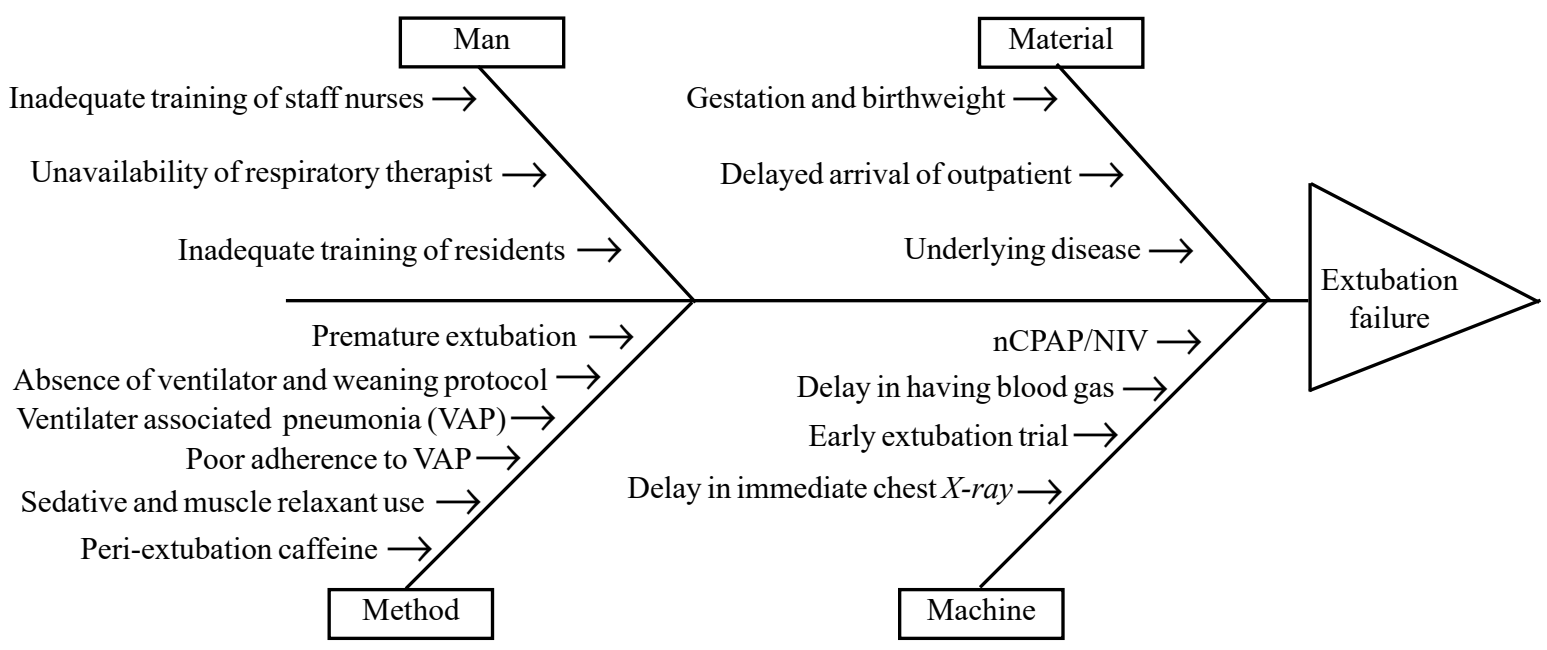

Fig. 1 Cause and effect diagram.

was to reduce extubation failure rate by $25 \%$ from baseline. Secondary outcomes to assess the impact of intervention were: time of first extubation attempt, multiple extubation failures (defines as two or more extubation failures in a newborn during hospital stay) and duration of mechanical ventilation. During the first PDCA cycle, patient data for all baseline and outcome variables were collected.

During PDCA cycle II, we uploaded the protocol in the hospital's digital document control system. Nursing staff and residents were given training monthly and ventilator and weaning strategies were discussed with them during daily rounds.

The study included all consecutively ventilated newborns during the study period. Newborns who died, discharged against medical advice (DAMA) or those referred to higher center before attempting first extubation were excluded from analysis.

Statistical analyses: SPSS 15 was used to analyze data. Data were examined for normality by KolmogorovSmirnov and Shapiro-Wilk tests. We used the chi-square test and Fischer's exact test for categorical variables. Independent t-test and Mann Whitney $U$ test were used for continuous variables, as appropriate. A $P$ value of $<0.05$ was considered statistically significant and all tests were 2-tailed. The data were analyzed on an intention-to-treat basis.

\section{RESULTS}

Study flow is depicted in Fig. 2. The demographic characteristics were similar in all three groups. In baseline period, PDCA-1 and PDCA-2, mean (SD) birth weight was 2168 (1008), 2176 (861.4), and 2226 (651) $\mathrm{g}$, and mean (SD) gestational age was 34.6 (3.4), 34.2 (4.6) and 35.0 (3.7) wk, respectively. In the three groups, males were $67 \%, 75 \%$ and $76 \%$ and inborn newborns were $61 \%, 42 \%$ and $53 \%$, respectively. Table I provide the details of outcomes in the three groups. There was a significant reduction in time to first extubation attempt.

Compliance to caffeine use, pre-extubation chest $X$-ray, target minimal ventilator settings, withholding enteral feed, inotropes and sedation according to extubation checklist was $100 \%$ in both post-protocol periods 1 and 2. Compliance to pre-extubation blood gas analysis was $33.3 \%$. All newborns were ventilated on either Synchronized intermittent mandatory ventilation pressure support or Assist control mode.

\section{Discussion}

In the present study, quality improvement PDCA cycles resulted in significant reduction in extubation failure rate. It also resulted in time to first extubation attempt.

The major constraint of this study is small sample size from a single center that limits generalizability and precludes subgroup analysis. The study group is heterogeneous in gestational age, birth weight and morbidities that can introduce selection bias. Prolonged mechanical ventilation in very preterm newborns could potentially confound the results. Out-born newborns coming late to our hospital preclude early surfactant therapy and/or early ventilator support. Our study did not measure other important outcomes e.g. retinopathy of prematurity, bronchopulmonary dysplasia and neurodevelopmental outcomes.

Extubation failure is multifactorial and a single 


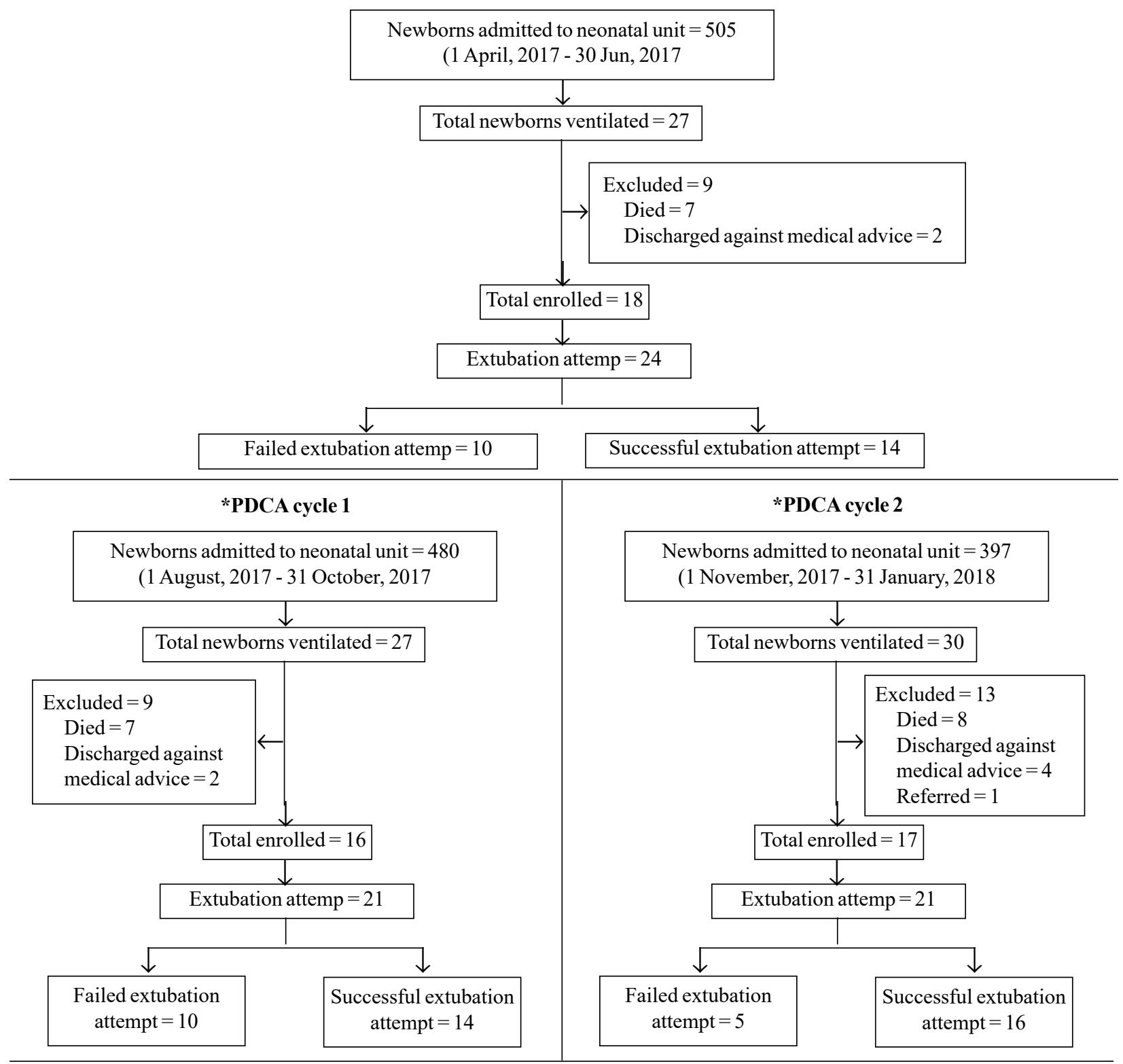

Fig. 2 Participant recruitment flow diagram.

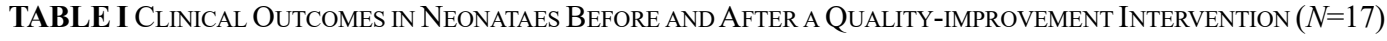

\begin{tabular}{lllr}
\hline Outcome & Pre-protocol period $(n=18)$ & PDCA-1 $(n=16)$ & PDCA-2 $(n=17)$ \\
\hline Extubation failure rate* & $10 / 24(41.7 \%)$ & $5 / 21(23.8 \%)$ & $5 / 21(23.8 \%)$ \\
Extubation failure $\geq 2^{\#} \mathrm{n} / \mathrm{N}(\%)$ & $4 / 18(22.2 \%)$ & $1 / 16(6 \%)$ & $0 / 17$ \\
Time (h) of first extubation attempt, median (IQR) & $71.5(46.5-75)$ & $51(16.6-92.4)$ & $38^{\$}(21.5-83)$ \\
Duration (h) of mechanical ventilation, median (IQR) & $73.5(70.5-139.3)$ & $68.5(18.8-96.3)$ & $49(22.0-112.5)$ \\
\hline
\end{tabular}

"PDCA: plan-do-check-act cycle; ${ }^{*}$ Number of re-intubations within 72 h of extubation $\div$ total number of extubation attempts (\%); ${ }^{*}$ Number of newborns needed $\geq 2$ reintubations within 72 h of extubation $\div$ total number of newborns in that period $(\%) ;{ }^{\$} P=0.046$ (pre-protocol vs PDCA-2).

intervention may not bring down its incidence. The extubation failure rate varies widely among centers depending on the characteristics of patients and the mode of support used after extubation. The definition of extubation failure varies widely in literature from 24 hours to 7 days after extubation $[6,9]$. Two randomized 


\section{What This Study Adds?}

- A ventilation and extubation strategy, implemented using the quality PDCA methodology, has the potential to reduce extubation failure and time to first extubation attempt in ventilated newborns.

controlled trials comprising a small subgroup of term newborns evaluating weaning protocol were inconclusive [8,10,11]. Hermato, et al. [12] demonstrated a significant reduction in extubation failure rate, the median age of first extubation attempt and the median duration of mechanical ventilation in newborns (birth weight $\geq 1250 \mathrm{~g}$ ) after implementing respiratory therapist-driven ventilation protocol in a retrospective study. These were similar to results of the present study.

Our findings underscore the importance of using quality improvement methodology $[13,14]$ in settings where failed extubation is a common troublesome phenomenon. The ventilation and extubation protocols can be further updated according to available resources at the local level as well as the current best scientific knowledge. Studies are needed to study the impact of this approach on other morbidities.

Acknowledgements: Dr Sudhir Mishra, Head, Department of Pediatrics, Tata Main Hospital for his valuable inputs in revising the manuscript. Air Marshal (Dr) Rajan Chaudhry, AVSM, VSM (Retd.), GM (Medical Services), Tata Main Hospital, for providing permission to submit the manuscript for publication. Contributors: RP: planning, collection and analyses of data, literature review and preparation of final manuscript; AKM: concept and design of the study, review and revision of manuscript, final approval of manuscript. Both authors approved the final version of manuscript, and are accountable for all aspects related to the study.

Funding: None; Competing Interest: None stated.

\section{REFERENCES}

1. Lemyre B, Davis Pg, De Paoli Ag, Kirpalani H. Nasal intermittent positive pressure ventilation (NIPPV) versus nasal continuous positive airway pressure (NCPAP) for preterm neonates after extubation. Cochrane Database Syst Rev. 2017;2:CD003212,

2. Ferguson KN, Roberts CT, Manley BJ, Davis PG. Interventions to improve rates of successful extubation in preterm infants: A systematic review and meta-analysis. JAMA Pediatr. 2017;171:165-74.

3. Al-Mandari H, Shalish W, Dempsey E, Keszler M, Davis
PG, Sant'anna G. International survey on periextubation practices in extremely preterm infants. Arch Dis Child Fetal Neonatal Ed. 2015;100:F428.

4. Shalish W, Anna GMS. The use of mechanical ventilation protocols in canadian neonatal intensive care units. Paediatr Child Health. 2015;20:e13-9.

5. Hiremath GM, Mukhopadhyay K, Narang A. Clinical risk factors associated with extubation failure in ventilated neonates. Indian Pediatr. 2009;46:887-90.

6. Giaccone A, Jensen E, Davis P, Schmidt B. Definitions of extubation success in very premature infants: A systematic review. Arch Dis Child Fetal Neonatal Ed. 2014;99:f124-7.

7. Hermeto F, Martins BMR, Ramos JRM, Bhering CA, Sant'anna GM. Incidence and main risk factors associated with extubation failure in newborns with birth weight $<1,250$ g. J Pediatr (Rio J). 2009;85:397-402.

8. Wielenga JM, van den Hoogen A, van Zanten HA, Helder $\mathrm{O}$, Bol B, Blackwood B. Protocolized versus non protocolized weaning for reducing the duration of invasive mechanical ventilation in newborn infants. Cochrane Database Syst Rev. 2016;3:cd011106.

9. Wang S-H, Liou J-Y, Chen C-Y, Chou H-C, Hsieh W-S, Tsao P-N. Risk factors for extubation failure in extremely low birth weight infants. Pediatr Neonatol. 2017;58: 145-50.

10. Schultz TR, Lin RJ, Watzman HM, Durning SM, Hales R, Woodson A, et al. Weaning children from mechanical ventilation: A prospective randomized trial of protocoldirected versus physician-directed weaning. Respir Care. 2001;46: 772-82.

11. G Randolph A, Wypij D, Venkataraman S, H Hanson J, Gedeit R, L Meert K, et al. Effect of mechanical ventilator weaning protocols on respiratory outcomes in infants and children: A randomized controlled trial. JAMA. 2002; 288:2561-8.

12. Hermeto F, Bottino MN, Vaillancourt K, Sant'anna GM. Implementation of a respiratory therapist-driven protocol for neonatal ventilation: Impact on the premature population. Pediatrics. 2009;123:e907-16.

13. Chawla D, Darlow BA. Development of quality measures in perinatal care - Priorities for developing countries. Indian Pediatr. 2018;9:797-802.

14. Mehta R, Sharma KA. Use of learning platforms for quality improvement. Indian Pediatr. 2018;9:803-8. 